\title{
Variations in social contexts and their effect on adolescent inhalant use: A latent profile investigation
}

\author{
Michael G. Vaughn $^{\mathrm{a}, *}$, Brian E. Perron ${ }^{\mathrm{b}}$, Matthew O. Howard ${ }^{\mathrm{b}}$ \\ ${ }^{a}$ University of Pittsburgh, School of Social Work and Center on Education and Drug Abuse Research, Pittsburgh, PA 15260, United States \\ ${ }^{\mathrm{b}}$ University of Michigan, School of Social Work and Department of Psychiatry, Ann Arbor, MI 48109, United States
}

Received 22 March 2007; received in revised form 16 May 2007; accepted 17 May 2007

\begin{abstract}
The social contexts surrounding the use and abuse of inhalants are poorly understood. The aim of this study was to utilize latent profile analysis (LPA) to identify specific subgroups of adolescents based on social contextual effects surrounding inhalant use episodes in a sample of 279 adolescent inhalant users. Findings revealed that a three-class solution exhibited the best empirical and conceptual fit with the data. Identified classes represented a gradient of low, moderate, and high levels of contextual effects where approximately one third of adolescent inhalant users reported high levels of inhalant use in response to social contextual influences. Subsequent validation analysis showed that these gradient-based classes were directly correspondent with severity in measures of psychopathology, past drug use, variety of inhalants used, and measures of impulsivity and fearlessness. Results indicate heterogeneity in contextual effects on inhalant use and suggest that follow-up studies should examine the role that susceptibility and exposure to contextual effects has on inhalant use.
\end{abstract}

(C) 2007 Elsevier Ireland Ltd. All rights reserved.

Keywords: Inhalants; Substance abuse; Latent profile analysis; Adolescent drug abuse

\section{Introduction}

Research consistently shows that social contextual influences are a strong motivating factor for experimenting with substances by children and adolescents, which can lead to problems of abuse and dependence. Generally, these contextual effects are conceptualized in the form of peer effect. A variety of methods have been used to measure peer effects, including the use of dilemmas of hypothetical characters (Brendt, 1979; Allen et al., 2006), best friend's substance use (Hussong and Hicks, 2003), the peer pressure inventory (Brown et al., 1986) and conformity scales (Santor et al., 2000). However, as reviewed by Kawaguchi (2004), many studies of peer effects assume the absence of contextual social effects. Others have also argued that peer effects in substance abuse research have been poorly conceptualized (e.g., Hussong and Hicks, 2003). The existing research on adolescent behavior, especially with respect to deviant behavior and substance use, has focused on behavioral similarity between individuals and their friends and peer groups (Kiesner et al.,

\footnotetext{
* Corresponding author.

E-mail address: mgv6@pitt.edu (M.G. Vaughn).
}

2004). However, as Kiesner et al. (2004) argued, much of the research has been conducted in the school context and typically examined only one relationship at a time. Additional research is needed to bring clarity to the conceptualization of peer influences and situating this line of research within the interpersonal environment, or social context, in which substances are used.

This study seeks to help fill these gaps in knowledge by describing and classifying the social contexts in which adolescents use substances, with a focus on inhalants. Prior research has suggested approximately that $9-20 \%$ of adolescents have engaged in huffing or sniffing inhalants such as gasoline, glue, shoe polish, paint, correction fluid, and other volatile organic compounds contained within widely available household products (Garofalo et al., 1999; Johnston et al., 2006; Wu et al., 2004). The social, physical, and cognitive consequences of abusing inhalants, along with the general absences of knowledge regarding the social context in which inhalants are used, also motivated the study of this specific type of substance. An important question that has yet to be addressed in the literature on inhalants is whether users are influenced by the same social pressures as found with other drugs of abuse? We explored this question by examining qualitative differences across social contexts by using latent profile analysis (LPA). This analytic strategy provided the 
basis for empirically deriving common or shared social contexts in which inhalants are used. The contexts identified were examined in relation to a variety of other clinical and behavioral measures to stimulate new conceptualizations related to peer context and substance use.

\section{Materials and methods}

\subsection{Sample and procedures}

The present study sample $(N=279)$ of inhalant users was drawn from a larger survey of the population of residents $(N=740)$ in the Missouri Division of Youth Services (DYS). Participation in the study was voluntary. The Missouri DYS is the legal guardian of all residents who are committed, for a variety of transgressions, to its care by the state's 45 juvenile courts. All youth providing written informed consent completed the structured face-to-face approximately 45-min interview assessing demographic characteristics, lifetime and annual use of inhalants, other drug use, substance-related problems, current and lifetime psychiatric symptoms, and externalizing behaviors. Study response rate was $97.7 \%$.

All interviewers completed an intensive 1-day training session and an interview editor was on-site at each facility as youth were interviewed to minimize interviewer omissions and errors. The sample recruitment protocol ensured that no youths who had completed the interview at one facility, then attempted to complete or were successful in completing the interview at another facility. This study was approved by DYS, the Washington University Human Studies Committee Institutional Review Board, the federal Office of Human Research Protection, and was granted a Certificate of Confidentiality by the National Institute on Drug Abuse (NIDA). Youth received \$10.00 for their participation.

The study sample had a mean age of 15.5 (S.D. $=1.13$ ). The majority was White $(74.7 \%)$, male $(84.3 \%)$, and $39.1 \%$ reported being from a household that received public assistance. Not surprisingly, the majority of the sample reported using alcohol (95.0\%) and marijuana (95.4\%).

\subsection{Measurement of social contexts}

The survey instrument contained a comprehensive assessment of the use of inhalants. This assessment included what inhalants were used and in what social contexts. There were nine survey items assessing different social contexts. Items were drawn and adapted from various questionnaires used in alcohol studies such as reasons for drinking questionnaire (RFDQ) (Zywiak et al., 1996). The scales were changed so they referred to inhalants rather than alcohol. Subjects responded to the items by indicating their level of agreement on how often they used inhalants for that particular context $(0=$ never, $3=$ almost always). These items were used to identify similar context across the subject pool. Internal consistency reliability for these nine items was .88 .

\subsection{Analytic strategy}

2.3.1. Latent profile analysis. As previously stated, LPA was used to identify the similar social contexts in which inhalants were used. Latent profile analysis is similar to latent class analysis (LCA), except LPA uses continuous or ordinal observed indicators whereas LCA analyzes categorical indicators. The underlying assumption of LPA is that the relationship among continuous indicators can be explained by a categorical latent variable. The continuous indicators are considered to be locally independent, meaning that the observed items are statistically independent within each latent class (Lazarfeld and Henry, 1968; Ruscio, 2004).

The assumption of local independence cannot be tested directly. However, it is established that the assumption can be violated when there is redundancy among the items- that is, asking the same question but using slightly different wordings (Byrne, 2005). Some of the nine items selected for the LPA in this study appeared to exhibit some conceptual overlap (see Table 1). For example, wanting to have a good time, to increase enjoyment, and to celebrate with friends are similar. An inspection of zero-order (Pearson-r) correlations among all the
Table 1

Summary of social contexts in which inhalants were used $(N=279)$

\begin{tabular}{lll}
\hline Effect & Mean & S.D. \\
\hline $\begin{array}{l}\text { Someone offered inhalants and felt } \\
\text { awkward refusing them }\end{array}$ & 0.89 & 0.99 \\
$\begin{array}{l}\text { Met with friends and wanted to have } \\
\quad \text { a good time }\end{array}$ & 1.20 & 1.07 \\
$\begin{array}{l}\text { With friends and suggested you go } \\
\quad \text { somewhere and use inhalants }\end{array}$ & 0.92 & 1.04 \\
$\quad \begin{array}{l}\text { Others in the same room were using } \\
\quad \text { inhalants and you felt expected to }\end{array}$ & 1.03 & 1.09 \\
$\quad$ join in & & \\
$\quad \begin{array}{l}\text { With friends and wanted to increase } \\
\quad \text { your enjoyment }\end{array}$ & 1.10 & 1.10 \\
$\quad \begin{array}{l}\text { Heard someone talking about their } \\
\text { past experiences with inhalants }\end{array}$ & 0.72 & 0.92 \\
$\begin{array}{l}\text { Pressured to use inhalants and felt } \\
\quad \text { you could not refuse }\end{array}$ & 1.69 & 1.40 \\
$\quad \begin{array}{l}\text { Wanted to celebrate with a friend } \\
\text { With a group of people and everyone } \\
\quad \text { was using inhalants }\end{array}$ & 0.65 & 0.97 \\
\end{tabular}

Note: range $=0-3$.

indicators was conducted. These correlations ranged ranged from .24 to .66. This suggested that there was some conceptual overlap, which is expected; however, there was no clear evidence of item redundancy. Moreover, Muthén considers collinearity among indicators to be a problem with extremely high correlations (Muthén, 2004a), and correlations around .60 should not present any problems (Muthén, 2004b).

Latent profile analysis was carried out using LatentGOLD (version 4.0) in an exploratory fashion. Specifically, rather than testing a class solution specified a priori, the fit of a series of different models was examined. A single-class model was examined first, and classes were added until no further improvements were observed. The empirical fit of the model was based on several fit indices including the Bayesian information criterion (BIC). An improvement in model fit results in a lower value on the BIC. Entropy was also examined, which is an index reflecting how well the indicators predict class membership and thus is indicative of class purity. Values closer to 1.0 indicate better prediction. Conditional bootstrap resampling procedures were also employed to evaluate final models. Conceptual fit of models is critical and was examined by using visual representations of the contexts to assess their interpretability and practical implications.

2.3.2. Comparative analysis. A comparative analysis of the different context derived from the LPA was conducted by testing their associations with demographic and clinical measures. This analysis was carried out using SPSS (version 14.0). Measures were selected based on prior empirical associations with substance use among adolescents. Specific demographic measures included gender (male/female) and receipt of public assistance (yes/no). Because DYS system youth are predominantly African-American and White Race was measured by collapsing categories to create a dichotomous measure (White/non-White). Location of residence prior to commitment was measured with four categories: urban city, suburban area, small town, and rural area.

Clinical measures for the comparative analysis included four subscales and the overall sum score from the brief symptom inventory (BSI) $(\alpha=0.96)$ : depression $(\alpha=0.83)$, anxiety $(\alpha=0.80)$, paranoid ideation $(\alpha=0.78)$, and psychoticism $(\alpha=0.80)$. A measure of externalizing behaviors was derived from the self report of delinquency $(\alpha=0.84)$, which ask youth if they have been involved in various forms of non-violent and violent antisocial acts. The personality traits of fearlessness (psychopathic personality inventory short form, $\alpha=0.70$ ) and impulsivity (antisocial process screening device, $\alpha=0.67$ ) were also assessed. Past year and lifetime drug use was indexed from an ordinal-like $(0=$ non-use to 3 = heavy use) matrix for 14 types of substances. From a set of 65 different types of inhalants, subjects were also queried about the different types of inhalants they used over their lifetime. 
Table 2

Fit Indices for latent classes defined by context of inhalant use

\begin{tabular}{llllll}
\hline No. of classes & LL & BIC (LL) & Npar & Class error & Entropy \\
\hline 1 & -3089.82 & 6331.69 & 27 & 0.00 & NA \\
2 & -2656.67 & 5521.68 & 37 & 0.02 & 0.92 \\
3 & -2570.90 & 5406.47 & 47 & 0.06 & 0.86 \\
4 & -2539.66 & 5400.31 & 57 & 0.09 & 0.81 \\
\hline
\end{tabular}

LL, log likelihood.

BIC, Bayesian information criterion.

Npar, number of parameters.

\section{Results}

\subsection{Descriptive summary}

Table 1 summarizes the contexts in which inhalants were used, ordered by the highest mean value of agreement. The most common contexts were "pressured to use and feeling an inability to refuse," followed by "met with friends and wanted to have a good time," and "with friends and wanted to increase your enjoyment." The least common context was "wanted to celebrate with a friend."

\subsection{Latent profile analysis}

A total of four LPA models were examined, ranging from one to four classes. Each model was estimated with 200 random starts, and no problems with local maxima were found. The empirical fit of the models and their estimated class sizes are summarized in Table 2. The one-class solution exhibited a poor fit with the data relative to the other models. The entropy values for all the models were very similar and greater than .80 , indicating that contextual effects for using inhalants were good predictors of class membership. Overall, the four-class solution exhibited the best empirical fit with the data based on the BIC. However, the improvement from the three-class to fourclass solution was negligible and entropy was greater for the three-class solution. Furthermore, conditional bootstrap resampling indicated that the three-class solution was the preferred fit $(-2 \log$ likelihood differential $=128.39, p<.0001)$ over the four-class alternative.

The conceptual fit of the models was examined through visual inspection. This involved plotting the estimated mean values for each social context effect by each class. Classes were clearly distinguishable and followed a severity gradient in terms of the susceptibility to social context effects ranging from low to medium to high susceptibility (see Fig. 1). Class 2 was clearly the most influenced followed by class 1 (moderate) and class 3 (least affected). There was also greater variability in mean values on the reasons compared to the four-class solution. The class sizes were also fairly balanced (see Table 3 ). A sensitivity analysis was performed to assess the stability of the three-class model. This involved allowing residuals of sets of indicators with the highest zero-order correlations to be freely estimated. No significant improvement in model fit or changes in the class structure were observed. Indicators with the highest zero-order correlations were also removed from the model, which resulted in a poorer model fit. Therefore, the three-class model with nine indicators and no correlated residuals was retained.

\subsection{Comparative analysis}

A comparative analysis of the three-class solution was conducted by examining the associations among the three classes with demographic and clinical factors. Chi-square tests revealed that class composition no differences by gender $\left(\chi^{2}[2]=0.36\right.$, $p=0.83$, receipt of public assistance $\left(\chi^{2}[2]=0.13, p=0.93\right)$, or urbanicity $\left(\chi^{2}[6]=7.00, p=0.32\right)$. A small difference was across clusters comparing White versus non-White subjects $\left(\chi^{2}[2]=0.13, p=0.04\right)$. Approximately $13 \%$ of Class 3 was comprised of White subjects, which was higher than their representation in Class 1 and Class 2 (6\% and 5\%, respectively).

One-way analysis of variance (ANOVA) with Dunnett's T3 and Bonferroni post hoc comparisons revealed significant dif-

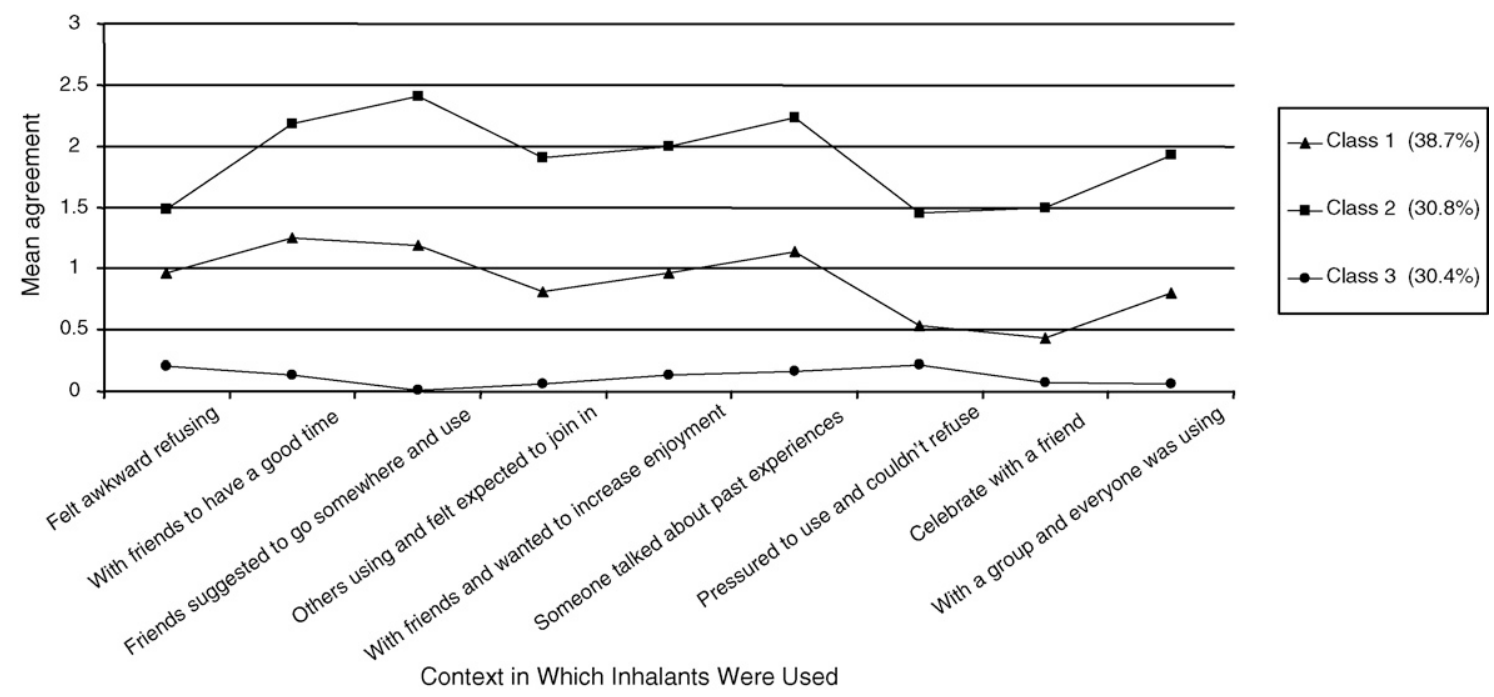

Fig. 1. Three-class solution of latent profile analysis representing mean agreement for indicators of social context. 
Table 3

Tests of clinical differences across three classes of inhalant users defined by typical contexts of inhalant use $(N=277)$

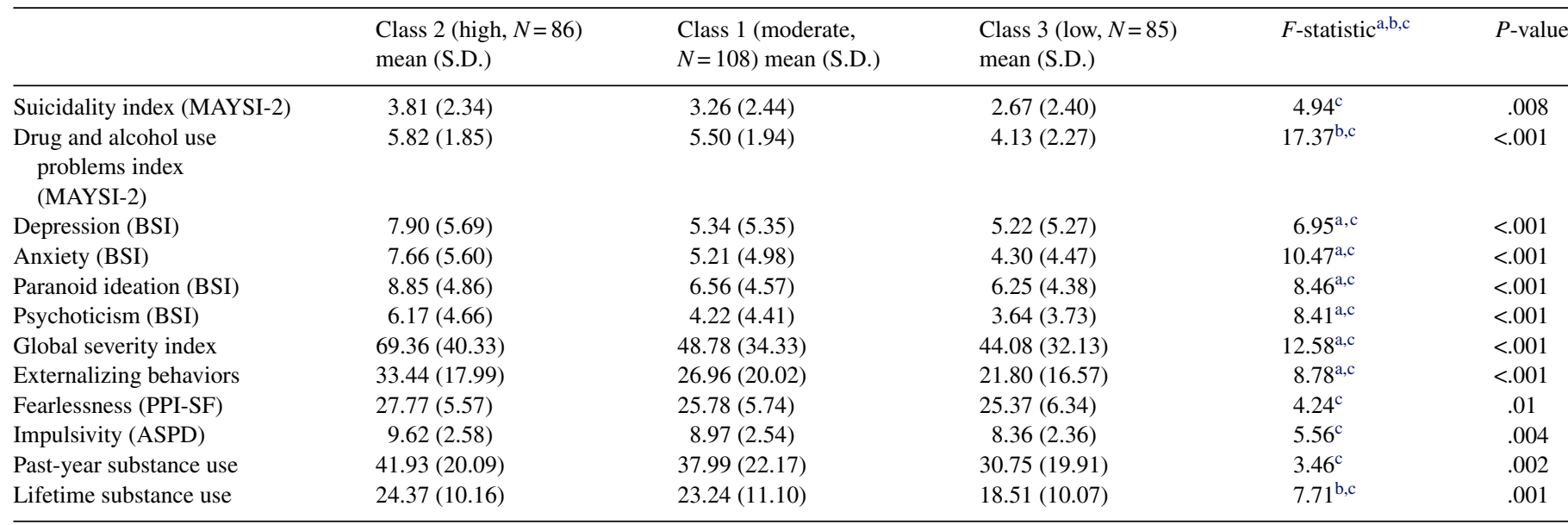

Note: Dunnett's T3 post hoc comparisons conducted for all ANOVAs.

${ }^{a}$ Classes 1 and 2 are different.

b Classes 2 and 3 are different.

c Classes 1 and 3 are different.

ferences on all the clinical variables (see Table 3). Class 2, which reported the highest level of contextual effects, also self-reported the highest levels across all of the clinical variables including fearlessness, impulsivity, and past year polysubstance use. Differences in mean levels for these variables were statistically significant compared to Class 3 (low contextual effects). Differences between the high contextual effects subgroup (Class 2) and the moderate subgroup were significant for all BSI subscales and total sum score $(p<.001)$.

We also examined the number of different types of inhalants used, which ranged from 1 to 16 . This was a count variable, so it exhibited a high skew $($ mean $=4.18$, S.D. $=3.04$, Skew $=1.05)$. Thus, it was dichotomized, reflecting one and more than one type of inhalant used over the lifetime. The classes showed significant differences on this measure $\left(\chi^{2}[2]=10.6, p=.005\right)$. Seventyseven percent of Class 1 subjects used more than one type of inhalant over the lifetime; among Class 2 subjects, 91\%; and Class 3 subjects, $52 \%$.

\section{Discussion}

Social contextual effects on inhalant users revealed a clear gradient-based class structure. Approximately one third of adolescent inhalant users reported a high number of different inhalants used in response to social contextual influences. These youth also scored high on clinical psychiatric symptom measures, suicide ideation, impulsivity, fearlessness, and other drug use including variety of inhalants. Adolescents in the low and moderate contextual effects classes self-reported lower mean levels across these same clinical variables. This, however, does not mean that these youth were free of psychopathology, only that indicators of high psychopathology went hand-in-hand with high levels of social contextual effects on use of inhalants. It is important to note that the measure of context may be confounded with frequency of inhalant use. It could be that the high effects class is simply using more in all contexts.
Although we are limited by the cross-sectional nature of the study design, we can speculate that there are at least two ways to interpret the present study findings. The first being that social contextual effects on adolescent inhalant use identified simply represents a continuum of susceptibility to the influences of others. Second, the variation in contextual effects is a result of differential exposure to social situations that condition the use of inhalants as well as other drugs. Given that the high effects class also scored high on indicators of impulsivity and fearlessness suggests that these traits may help propel them toward sensation seeking activities with others. Building on our findings, future studies will need to examine the stability of micro contextual effects on identified classes and closely assess the relationship between susceptibility/exposure of social influence on patterns of initiation, escalation, and cessation of inhalant use.

Although causal influences around use cannot be derived in this study, the comparative analysis suggests various possibilities. For example, it is possible that social pressures lead to greater quantities of use, thereby explaining the linear association between contextual influences and degree of clinical and behavioral problems. It is also possible that higher levels of psychopathology also impede problem solving and coping skills behavior. This would suggest that adolescents with higher levels of psychopathology are more susceptible to social influences.

There has been little systematic exploration of the underlying heterogeneity of inhalant users. Present study findings show that inhalant user's are indeed subject to the effects of social influences as seen with other adolescent drug abusers. Further, social contextual effects can be differentiated by relatively homogeneous subgroups of users that reflect a severity continuum. This continuum may simply reflect high drug use of all kinds and the co-occurring psychological distress that accompanies this pattern. Also, experimental users in the low effects group perhaps are enmeshed in a peer network that not only does not use inhalants but also is less likely to be regular drug users generally. Although neuroscience research on inhalants has shown that 
reinforcing effects operate via GABA and dopaminergic mechanisms (Ridenour, 2005), these mechanisms also occur within a larger context of social influence. The link between the biological and social provides an interesting social neuroscience avenue for future research on inhalant abuse. Future studies should be designed so that temporal ordering and the interaction effects of these variables will allow for firmer determinations to be made.

Examining inhalant use within a social context also has important service implications. For example, this knowledge could help treatment providers create simulations that closely reflect real world situations in which adolescents may be particularly susceptible to substance use. Items contained within Table 1 from this study could be used to guide these types of efforts. This would enhance the opportunity to practice coping behaviors and refusal strategies. By knowing the different contexts in which substances are used, prevention efforts (e.g., social marketing) can be more effectively targeted.

\section{Acknowledgments}

Grateful acknowledgements to Mark Steward, Director, Gail D. Mumford, Deputy Director, and Alicia Jenkins, Dual Jurisdiction/Case management Coordinator of the Missouri Division of Youth Services. Funding for this study was provided by NIDA Grant DA 15929 and DA 15556; NIDA had no further role in study design; in the collection, analysis and interpretation of data; in the writing of the report; or in the decision to submit the paper for publication.

Contributors: The first two authors jointly conceptualized the study. Michael Vaughn led the statistical analyses and writing. Brian Perron assisted with all the analyses and shared in the writing. Matthew Howard edited and contributed to secondary drafts. All authors contributed to and have approved the final manuscript.

Conflict of interest: None declared.

\section{References}

Allen, J.P., Porter, M.R., McFarland, F.C., 2006. Leaders and followers in adolescent close friendships: susceptibility to peer influence as a predictor of risky behavior, friendship instability, and depression. Dev. Psychopathol. 8, $155-172$.

Brendt, T.J., 1979. Developmental changes in conformity to peers and parents. Dev. Psychopathol. 15, 606-616.

Brown, B.B., Clasen, D.R., Eicher, S.E., 1986. Perceptions of peer pressure, peer conformity dispositions, and self-reported behavior among adolescents. J. Pers. Soc. Psychol. 22, 521-530.

Byrne, B.M., 2005. Factor analytic models: viewing the structure of an assessment instrument from three perspectives. J. Pers. Assess. 85, 17-32.

Garofalo, R., Wolf, R.C., Wissow, L.S., Woods, E.R., Goodman, E., 1999. Sexual orientation and risk of suicide attempts among a representative sample of youths. Arch. Pediatr. Adolesc. Med. 153, 487-493.

Hussong, A.M., Hicks, R.E., 2003. Affect and peer context interactively impact adolescent substance use. J. Abnorm. Child Psychol. 31, 413426 .

Johnston, L.D., O’Malley, P.M., Bachman, J.G., Schulenberg, J.E., 2006. Monitoring the Future National Survey Results on Drug Use, 1975-2005, vol. I, Secondary school students (National Institute of Drug Abuse, NIH Publication No. 06-5883) Bethesda, MD.

Kawaguchi, D., 2004. Peer effects on substance use among American teenagers. J. Popul. Econ. 17, 351-376.

Kiesner, J., Kerr, M., Stattin, H., 2004. "Very Important Persons" in adolescence: going beyond in-school, single friendships in the study of peer homophily. J. Adolesc. 27, 545-560.

Lazarfeld, P.F., Henry, N.W., 1968. Latent Structure Analysis. Houghton Mifflin, Boston.

Muthén, L., 2004a. Latent profile analysis. Message posted to StatModel online forum, archived at http://www.statmodel.com/discussion/messages/ 13/115.html?1175697157 (accessed on May 21, 2007).

Muthén, L., 2004b. Latent class analysis and cluster analysis. Message posted to StatModel online forum, archived at http://www.statmodel. com/discussion/messages/13/155.html?1141671585 (accessed on May 21, 2007).

Ridenour, T.A., 2005. Inhalants: Not to be taken lightly anymore. Curr. Opin. Psychiatry 18, 243-247.

Ruscio, J., 2004. A nontechnical introduction to the taxometric methods. Understanding Statistics 3, 151-194.

Santor, D.A., Messervey, D., Kusumakar, V., 2000. Measuring peer pressure, popularity, and conformity in adolescent boys and girls: predicting school performance, sexual attitudes, and substance abuse. J. Youth Adolesc. 29, 163-182.

Wu, L., Pilowsky, D.J., Schlenger, W.E., 2004. Inhalant abuse and dependence among adolescents in the United States. J. Am. Acad. Child Adolesc. Psychiatry $43,1206-1214$

Zywiak, W.H., Connors, G.J., Maisto, S.A., Westerberg, V.S., 1996. Relapse research and the reasons for drinking questionnaire: a factor analysis of Marlatt's relapse taxonomy. Addiction 91, 121S-130S. 